\title{
METASTABILITY OF NON-UNIFORM SYSTEMS
}

\author{
Onno L.J. GIJZEMAN and Hendrik N.W. LEKKERKERKER ₹ \\ lan 't Hoff Lahorator.' Unircrsity of Utrecht, Padualaan 8,3584 CH Utrecht, The Netherlands
}

Received 22 November 1982

A simple theory is given, based on the nearest-neighbour regular solution model, that predicts that below a certain temperature non-uniform concenitation profiles can be metastable in binay mixtures. The agreenent with recently obtained experimental data is satisiactory.

\section{Introduction}

A theoretical treatment of non-uniform systems and its application to the spinodal decomposition of binary mixtures has been formulated by Cahn and coworkers $[1-3]$. In this ireatment a non-uniform systim reaches equilibrium via a diffusion process against the concentration gradient. In the final state the syscon consists of two honogeneous plases in which no concentration gradients are present. However, in a recent study in this laboratory Mesters et al. [4] found extensive concentration gradients of nickel in copper single crystals after thermal decomposition of $\mathrm{Ni}(\mathrm{CO})_{4}$ at the copper surface. An illustrative example of their results is presented in fig. 1 . As expected on the basis of interfacial energy considerations, virtually no nickel is present at the copper surface. but its concentration rises steeply inside the crystal and extends over wughly 1000 atomic layers. This profile was found to be stable up to about $400^{\circ} \mathrm{C}$ aithough the profile flatched with increasing temperature.

In this letter we put forward a simple theoretical explanation for the observed effects based on the nearest-neighbour regular solution model (Bragg Williams or mean field approximation $[5,6])$. It is shown that below a certain temperature a concentrafion profile of the form found experimentally is in fiet metastable.

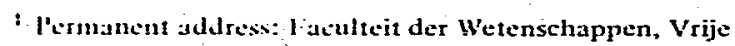
Iniversiteit lirusiel. 1050 Brussels. Belgium.

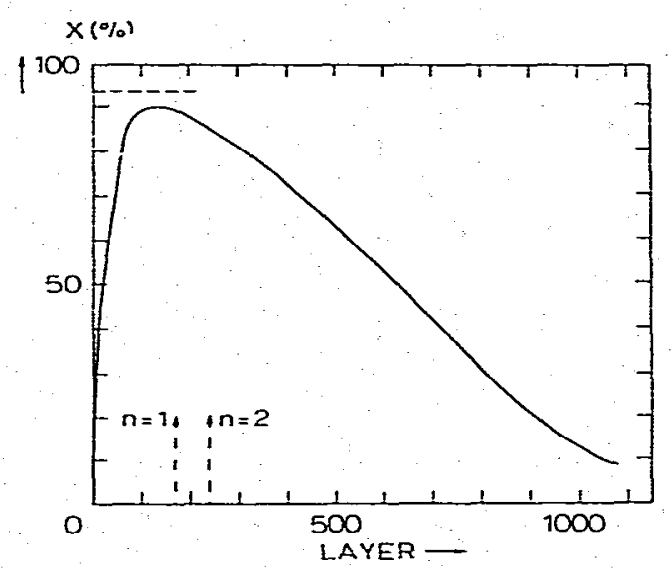

Fig. 1. Nickel fraction as a function of layer number for the Cu(110) surface following thermal decomposition of $\mathrm{Ni}(\mathrm{CO})_{4}$. After ref. 14$]$.

\section{Theory}

The free energy per unit volume of mixing of $N_{\mathrm{A}}$ atoms $A$ and $N_{\mathrm{B}}$ atoms $\mathrm{B}$ in the nearest-neighbour regular solution model is given by

$$
\begin{aligned}
f= & \rho\{\gamma \Omega x(1-x) \\
& \left.+k_{\mathrm{B}} T[x \ln x+(1-x) \ln (1-x)\}\right\} .
\end{aligned}
$$

Here $\rho$ is the number of atoms per unit volume, $x$ the mole fraction of atoms $B, \gamma$ the number of nearest neighbours and $\Omega 2$ the weighted difference of the A- 
$A, B-B$ and $A-B$ bond strengths,

$\Omega=V_{\mathrm{AB}}-\frac{1}{2}\left(V_{\mathrm{AA}}+V_{\mathrm{BB}}\right)$.

Assuming a non-uniform concentration profile of the form

$x(z)=A z \mathrm{e}^{-z / \xi}$

with $\xi$ much larger than the distance $d$ between the layers one finds for the free energy of mixing per unit area of surface:

$$
\begin{gathered}
F / k_{\mathrm{B}} T=\left(1 / k_{\mathrm{B}} T\right) \int_{0}^{\infty} f[x(z)] \mathrm{d} z \\
=\rho A \xi^{2}\left[\frac{\gamma \Omega}{k_{\mathrm{B}} T}\left(1-\frac{1}{4} y\right)+\ln y\right. \\
\left.\quad+\sum_{k=1}^{\infty} \frac{(k-1) !}{(k+1)^{k+2}} y^{k}-C-2\right],
\end{gathered}
$$

where $C=0.577125 \ldots$ is Eulers constant and $y=A \xi$.

Here the Cahn-Hilliard gradient term $K(\mathrm{~d} x / \mathrm{d} z)^{2}$, with $K \approx \gamma \Omega d^{2}$ has been neglected. This is justified for the non-uniformity in the concentration we are considering here, since it has a length scale $\xi \gg d$ and thus the contribution of the gradient term is $\gamma \Omega(d / \xi)^{2} \ll \gamma \Omega$.

The concentration profile (3) has a maximum

$x_{\max }=A \xi / \mathrm{e}=y / \mathrm{e}$

at

$z_{\max }=\xi$

Since physically $x \leqslant 1$ it follows from eq. (5) that $y \leqslant$ $e$. The total number of penetrated nickel atoms per unit area is given by

$\Gamma=\rho \int_{0}^{\infty} x(z) \mathrm{d} z=\rho A \xi^{2}$.

From eqs. (4) and (7) it is clear that the mixing free energy is proportional to the amount of nickel dissolved and further depends on the form of the non-uniform concentration profile only through the parameter $y$. Extrema of $F$ for a fixed amount of dissolved amount nickel are found from

$\frac{1}{4} \gamma \Omega / k T=\sum_{k=0}^{\infty}\left[k 1 /(k+1)^{k+2}\right] y^{k-1}$

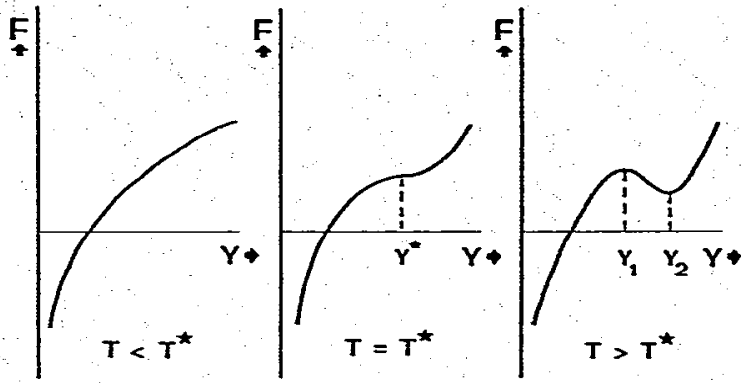

Fig. 2. Schematic representation of the behaviour of the free energy $(F)$ as a function of the profile parameters $y=A \xi$, for $T<T^{*}$ (left-hand figure); $T=T^{*}$ (middle figure) and $T>$ $T^{*}$ (right-hand figure).

Numerically we find from eq. (8):

(a) $k_{\mathrm{B}} T / \gamma \Omega>0.354 ; F$ has no extrema.

(b) $k_{\mathrm{B}} T / \gamma \Omega=k_{\mathrm{B}} T^{*} / \gamma \Omega=0.354 ; F$ has an in-

flection point for $y=y^{*}=2.29$. This corresponds to a distribution with a maximum $x_{\max }=y * / e=0.842$ at $z_{\max }=\Gamma / \rho y^{*}=0.44 \Gamma / \rho$.

(c) $k_{\mathrm{B}} T / \gamma \Omega<0.354 ; F$ has a maximum for $y_{1}<$ $y^{*}$ and a minimum for $y_{2}>y^{*}$.

The behaviour of $F$ is illustrated schematically in fig. 2. Note that the lowest value of $F$ is reached for $y \rightarrow 0$, which corresponds to the limit of an uniform distribution. From the results above it appears that for temperatures $T<T^{*}$ (which in turn lies below the critical demixing temperature, $k_{\mathrm{B}} T_{\mathrm{c}} / \gamma \Omega=\frac{1}{2}$ ) a non-uniform distribution is metastable.

In order to check to what extent this conclusion depends on the chosen form of the concentration profile we repeated the above calculations for profiles of the form

$x(z)=A z^{n} \mathrm{e}^{-2 / 5}, \quad n=2,3, \ldots$.

The free energy is then given by

$$
\begin{aligned}
& \frac{F}{k_{\mathrm{B}} T}=\Gamma\left[\frac{\gamma \Omega}{k_{\mathrm{B}} T}\left(1-y \frac{(2 n) !}{n ! 2^{2 n+1}}\right)\right. \\
& \quad+\sum_{k=1}^{\infty} \frac{[n(k+1)] !}{n ! k(k+1)^{k n+n+2}} y^{k+\ln y} \\
& \left.\quad+n\left(1+\frac{1}{2}+\frac{1}{3}+\ldots+1 / n-C\right)-n-2\right],
\end{aligned}
$$

where $y=A \xi^{n}$ and $\Gamma=\rho n ! A \xi^{n+1}$. The free energy given by. eq. (9) has qualitatively the same form as the previous expression. The extrema of $F$ are now found from 
$\frac{\gamma \Omega}{k_{\mathrm{B}} T} \frac{(2 n) !}{n ! 2^{2 n+1}}=\sum_{k=0}^{\infty} \frac{[n(k+1)] !}{n !(k+1)^{k n+n+2}} y^{k-1}$

For $n=2$ one finds $k_{\mathrm{B}} T^{*} / \gamma \Omega=0.357$ for $y^{*}=1.545$ corresponding to a distribution with a maximum $x_{\max }=4 y^{*} / e^{2}=0.839$ at $z_{\max }=\Gamma / \rho y^{*}=0.65 \Gamma / \rho$. Thus the value of the maximum is slightly lower and its position is somewhat further from the surface. but qualitatively the results are the same.

\section{Discussion}

As has been shown in section 2 a concentration profile of the form $A z \exp (-z / \xi)$ is metastable below a temperature $T^{*}$, equal to $0.354 \gamma \Omega / k_{\mathrm{B}}($ for $n=1)$ or $0.357 \gamma \Omega j k_{\mathrm{B}}($ for $n=2)$. The difference between the (local) minimum and the maximum value of $F$ depends of course on $T^{*}-T$. For $T=0.99 T^{*}$ one finds from eq. (S) for the profile $A z \exp (-z / \xi)$ that the maximum is located at $y_{1}=2.12$ and the minimum at $y_{2}=2.43$. The concentration profiles for these two cases are shown in fig. 3 , for a total number

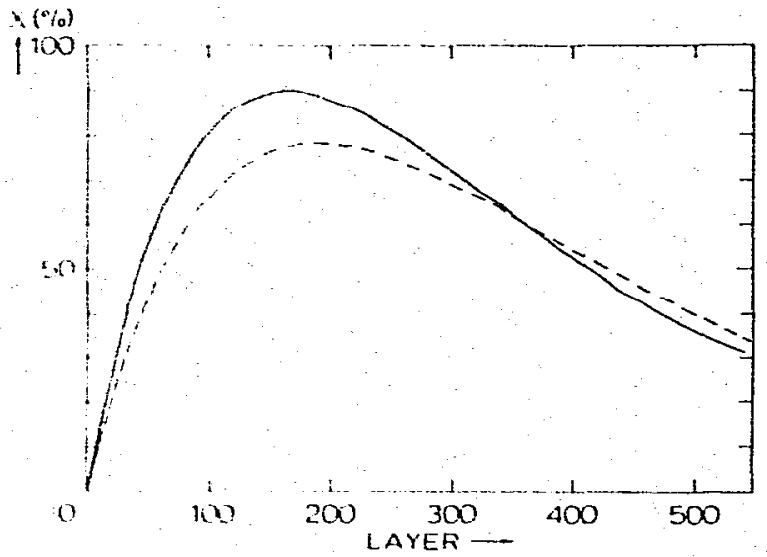

1. it.. 3. Calculated comentation profiles corresponting to the lusal minimum ( . ...) and the maximum (-..-) of the frec encigy. l'atameters as described in text. of penetrated nickel atoms of 400 per surface unit cell, which is roughly the experimental value deduced from fig. 1 . The profiles are seen to be rather different. The difference in free energy per penetrated nickel atom in this case is from eq. (4) $F / \Gamma \approx 0.005 k_{\mathrm{B}} T$. Taking into account that in order to change the profile at a surface unit cell a number of 400 atoms must participate collectively, one obtains an energy barrier of $2 k_{\mathrm{B}} T$ for this process. The stability of the nonuniform profile increases with increasing $T^{*}-T$ for example for $T=0.95 T^{*}$ one finds an energy difference of $5 k_{\mathrm{B}} T$ per surface unit cell.

We now turn our attention to the experimental data displayed in fig. 1 , taken at $200^{\circ} \mathrm{C}$. In order to evaluate $k_{\mathrm{B}} T / \gamma \Omega$ we use the mean field relation $k_{\mathrm{B}} T_{\mathrm{c}} / \gamma \Omega=0.5$ with $T_{\mathrm{c}}=700 \mathrm{~K}$ [7]. For $T=200^{\circ} \mathrm{C}$ one then obtains $k_{\mathrm{B}} T / \gamma \Omega=0.34$. For the profile with $n=1$ this implies a maximum nickel fraction of $93 \%$ at layer 160 . For the profile with $n=2$ the corresponding values are $94 \%$ at layer 230 . Both sets of values are indicated in fig. 1 and are seen to be qualitatively and even seni-quantitatively in agreement with the experimental data.

In summary we conclude that our approach predicts that non-uniform concentration profiles might be metastable below a certain temperature. The experimental results are well described by our theory.

\section{References}

|1| J.W. Calun and J.E. Hilliard. J. Chem. Phys. 28 (1958) 258.

[2] J.W. Cahn. Trans. AlME 242 (1968) 166.

[3] J.1. Hilliard, in: Phase transformations (Am. Soc. Metals. Metals Park, Ohio, 1970) p. 497

14] C.M.A.M. Afesters et al., to be published.

[5] E.A. Guggenheim, Mixtures (Oxford Univ. Press, London. 1952).

[6] R.A. Swalin. Themodynamics of solids (Wiley, New York. 1972).

17| P.E.C. Pranken and V. Ponec, J. Catal. 42 (1976) 398; J. Vrijen, Thesis, Utrecht (1977):

J. Aalders. Thesis. Utrecht (1982). 